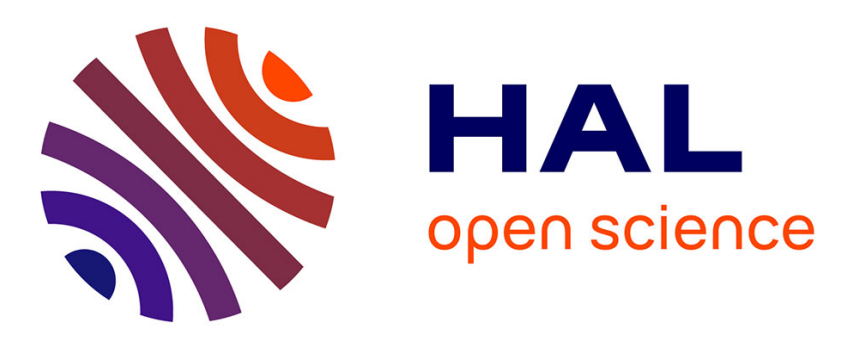

\title{
Broadband enhancement and inhibition of single quantum dot emission in plasmonic nano-cavities operating at telecommunications wavelengths
}

David Elvira, Remy Braive, Grégoire Beaudoin, Isabelle Sagnes, Jean-Paul Hugonin, Izo Abram, Isabelle Robert-Philip, Philippe Lalanne, Alexios Beveratos

\section{To cite this version:}

David Elvira, Remy Braive, Grégoire Beaudoin, Isabelle Sagnes, Jean-Paul Hugonin, et al.. Broadband enhancement and inhibition of single quantum dot emission in plasmonic nano-cavities operating at telecommunications wavelengths. Applied Physics Letters, 2013, 103, pp.061113. 10.1063/1.4818131 . hal-00859960

\section{HAL Id: hal-00859960 \\ https://hal-iogs.archives-ouvertes.fr/hal-00859960}

Submitted on 16 Nov 2015

HAL is a multi-disciplinary open access archive for the deposit and dissemination of scientific research documents, whether they are published or not. The documents may come from teaching and research institutions in France or abroad, or from public or private research centers.
L'archive ouverte pluridisciplinaire HAL, est destinée au dépôt et à la diffusion de documents scientifiques de niveau recherche, publiés ou non, émanant des établissements d'enseignement et de recherche français ou étrangers, des laboratoires publics ou privés. 


\section{AIP Applied Physics Letters}

\section{Broadband enhancement and inhibition of single quantum dot emission in plasmonic nano-cavities operating at telecommunications wavelengths}

David Elvira, Rémy Braive, Grégoire Beaudoin, Isabelle Sagnes, Jean-Paul Hugonin, Izo Abram, Isabelle RobertPhilip, Philippe Lalanne, and Alexios Beveratos

Citation: Applied Physics Letters 103, 061113 (2013); doi: 10.1063/1.4818131

View online: http://dx.doi.org/10.1063/1.4818131

View Table of Contents: http://scitation.aip.org/content/aip/journal/apl/103/6?ver=pdfcov

Published by the AIP Publishing

\section{Articles you may be interested in}

Enhanced single photon emission from positioned InP/GalnP quantum dots coupled to a confined Tammplasmon mode

Appl. Phys. Lett. 106, 041113 (2015); 10.1063/1.4907003

Spontaneous emission and collection efficiency enhancement of single emitters in diamond via plasmonic cavities and gratings

Appl. Phys. Lett. 103, 161101 (2013); 10.1063/1.4817397

Quantum-dot nano-cavity lasers with Purcell-enhanced stimulated emission Appl. Phys. Lett. 100, 131107 (2012); 10.1063/1.3697702

Room temperature spontaneous emission enhancement from quantum dots in photonic crystal slab cavities in the telecommunications $C$ band Appl. Phys. Lett. 94, 123101 (2009); 10.1063/1.3104855

Enhanced spontaneous emission rate from single InAs quantum dots in a photonic crystal nanocavity at telecom wavelengths

Appl. Phys. Lett. 91, 123115 (2007); 10.1063/1.2789291

\section{AIP $\left.\right|_{\text {APL Photonics }}$}

APL Photonics is pleased to announce Benjamin Eggleton as its Editor-in-Chief

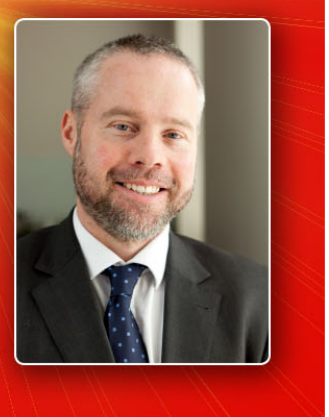




\title{
Broadband enhancement and inhibition of single quantum dot emission in plasmonic nano-cavities operating at telecommunications wavelengths
}

\author{
David Elvira, ${ }^{1}$ Rémy Braive, ${ }^{1}$ Grégoire Beaudoin, ${ }^{1}$ Isabelle Sagnes, ${ }^{1}$ Jean-Paul Hugonin, ${ }^{2}$ \\ Izo Abram, ${ }^{1}$ Isabelle Robert-Philip, ${ }^{1, a)}$ Philippe Lalanne, ${ }^{2}$ and Alexios Beveratos ${ }^{1}$ \\ ${ }^{1}$ Laboratoire de Photonique et Nanostructures LPN-CNRS UPR20, Route de Nozay, 91460 Marcoussis, \\ France \\ ${ }^{2}$ Laboratoire de Photonique, Numérique et Nanosciences, Institut d'Optique, Univ. Bordeaux 1, CNRS, \\ 33405 Talence cedex, France
}

(Received 19 June 2013; accepted 25 July 2013; published online 7 August 2013)

\begin{abstract}
A plasmonic microcavity providing broadband control of spontaneous emission for large and sparse semiconductor quantum dots emitting at telecommunications wavelengths is proposed. By designing and fabricating such a cavity, we demonstrate a broadband Purcell effect with spontaneous emission enhancement over a broad spectral range of $\Delta \lambda \simeq 20 \mathrm{~nm}$ with a 3.9-fold maximum enhancement, as well as inhibition over $\Delta \lambda \simeq 100 \mathrm{~nm}$ around $1.3 \mu \mathrm{m}$. The broadband feature relaxes the constraint on spectral matching between the dot emission and the cavity mode, favourable for implementing efficient non-classical light sources or nanoscale lasers. (C) 2013 AIP Publishing LLC.

[http://dx.doi.org/10.1063/1.4818131]
\end{abstract}

The extreme confinement of the electromagnetic field in nanoscale optical cavities, whose volume is of the order of a cubic wavelength, modifies significantly the radiative properties of emitting dipole. In particular, it may give rise to enhancement or inhibition of the spontaneous emission rate with respect to its value in free space, usually referred to as the Purcell effect. ${ }^{1}$ First observed in atomic systems, ${ }^{2}$ this effect was later implemented on ensembles of solidstate emitters ${ }^{3}$ and then on single emitters embedded in different types of low-loss dielectric cavities. ${ }^{4-7}$ Enhancement of spontaneous emission is now at the heart of a wide variety of potential applications, ranging from the production of quantum states of $\operatorname{light}^{8-11}$ to low-threshold microcavity lasers. ${ }^{12-14}$ In order to be viable, however, most of these applications require sources operating at telecommunications wavelengths particularly around $1.3 \mu \mathrm{m}$ and $1.5 \mu \mathrm{m}$. The Purcell effect in this wavelength range has recently been observed by embedding large semiconductor quantum dots (QDs) and dashes in dielectric cavities. ${ }^{15,16}$ However, significant Purcell enhancement of spontaneous emission requires simultaneously good spectral and spatial matching between the emitter and a narrow-bandwidth microcavity mode, requirements that are often compromised by the random distribution in space, spectrum and polarization of most types of solid-state emitters. One approach to overcoming this problem and optimizing spectral and spatial matching is to place the emitter deterministically in the cavity either by identifying its position and emission wavelength and then building the cavity around it, ${ }^{7,17}$ or by controlling its growth site. ${ }^{18,19}$ Another strategy exploits the weak sensitivity to spectral matching in low-Q plasmonic nano-cavities or nano-antennas in which the emitting dipole transfers the excitation to the surface plasmons of the metallic nanostructure, which can then radiate. Various metallic structure geometries coupled to solid-state emitters radiating below $1 \mu \mathrm{m}$ have already been implemented. ${ }^{20-22}$

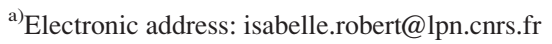

However, while these structures relax the constraint on spectral matching, their small dimensions present an engineering challenge in adequately positioning single emitters within the evanescent field of the plasmons. This difficulty is compounded when the emitters cannot be considered to be point-like, as is the case of QDs or dashes emitting at telecommunications wavelengths. The lateral dimensions of such QDs are of a few tens of nanometers ${ }^{23}$ and thus are illsuited to be embedded in ultimately-small plasmonic nanocavities, whose radius is of the order of $50 \mathrm{~nm}$. In addition, the small volume of such nanocavities is a disadvantage when the emitter density is very low, since the probability of having an emitter inside such a nanocavity becomes negligible.

In this work, we propose a cavity geometry suitable for operation with large and sparse emitters. This cavity is derived from the plasmonic nano-cylinder architecture, by engineering the two parameters that determine the value of the Purcell factor: the volume and the quality factor. The emitter is embedded in a small semiconductor cylinder which is inserted in a metallic blind hole, so that photons escape through the aperture of the hole. Given the typical dimensions and densities of self-assembled semiconductor QDs, the cylindrical cavity should have a radius of a few hundred nanometers (of the order of the optical wavelength in the semiconductor) in order to accommodate the large and relatively sparse QDs. For such a large radius the reflectivity of the blind hole aperture is very low $(\sim 30 \%$, equivalent to that of the semiconductor-air interface), thus lowering the quality factor of the cavity. This latter quantity may be restored by extending the metallic sheath, so that the metal-coated semiconductor cylinder is surmounted by an air-filled metallic collar (see Fig. 1). As the collar diameter is smaller than the half-wavelength of light in air, it constitutes a pinhole that does not support propagative electromagnetic modes and thus acts as a mirror that increases the quality factor, while at the same time it serves as the output coupler of the cavity. By measuring the emission dynamics of self-assembled InAsP 
QDs embedded in a metallic cavity based on this modified geometry, we demonstrate broadband spontaneous emission enhancement over a spectral range of $20 \mathrm{~nm}$ at $1.3 \mu \mathrm{m}$ and robust to spatial mismatch, as well as inhibition over a spectral range of $100 \mathrm{~nm}$.

The hybrid semiconductor-metal nanostructure under investigation is a cylindrical indentation of depth $600 \mathrm{~nm}$ and radius $400 \mathrm{~nm}$ in a sheet of gold. It is filled with a $260 \mathrm{~nm}$ high InP semiconductor nano-cylinder, so that the top $340 \mathrm{~nm}$ of the indentation are empty (see Fig. 1 Top). This structure presents a resonance at $\lambda=1325 \mathrm{~nm}$ with a full-width at halfmaximum (FWHM) of $20 \mathrm{~nm}$ corresponding to a quality factor of $Q=60$ (see Fig. 1 Bottom). Simulation of its modal structure indicates that this resonance corresponds to the third-order mode with modal volume of the order of $0.5(\lambda / n)^{3}$, where $n$ is the refractive index of the semiconductor; the distribution of the field inside the cavity is maximum at the center and decreases towards the periphery, while displaying two nodes radially. The central plane of the InP nanocylinder incorporates a single layer of InAsP QDs with average lateral dimensions of $40 \mathrm{~nm}$ and density $\sim 100 \mathrm{QD} / \mu \mathrm{m}^{2}$, so that each cavity contains on average $\sim 50$ QDs. At temperatures of $4 \mathrm{~K}$ and at low excitation powers the emission spectrum of the QD ensemble consists of a series of discrete peaks, each corresponding to the exciton transition of a QD, with a distribution centered around $\lambda \simeq 1.5 \mu \mathrm{m}$ and a FWHM of $100 \mathrm{~nm} .{ }^{24}$ Thus, only one or two QDs in the cavity are spectrally matched with the cavity mode at $1325 \mathrm{~nm}$. Under high excitation powers, on the other hand, the emission spectrum of each QD becomes very broad, due to the multiple electron-hole pairs present in it, ${ }^{24}$ and extends throughout the $1300 \mathrm{~nm}$ region. It thus acts as a "white intra-cavity lamp" allowing us to obtain the spectrum of the cavity, shown on Fig. 1 (bottom)-black curve.

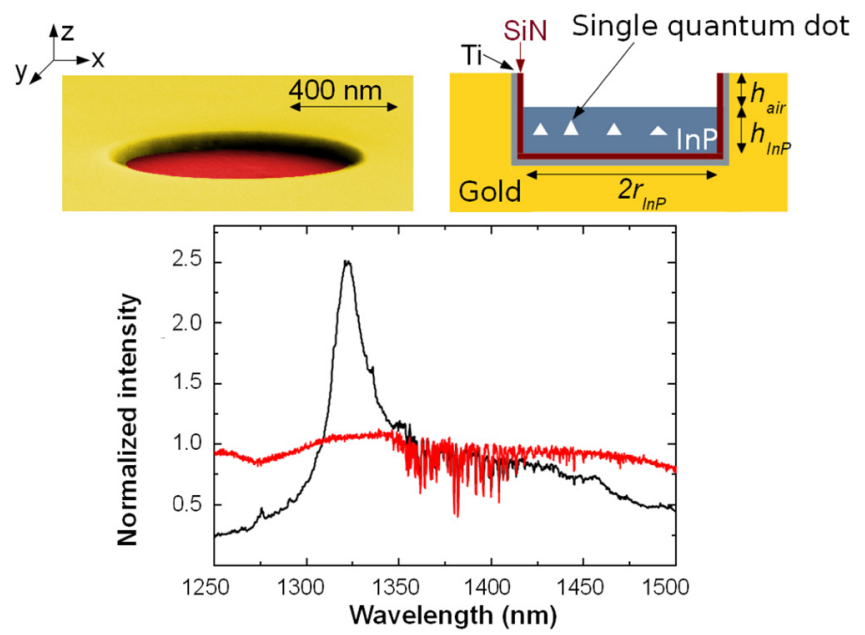

FIG. 1. (Top left) Scanning electron microscope image of a gold cavity with air-filled collar. (Top right) Cross-section of the structure along the $x z$ plane; the emitting dipoles, placed in the central plane of the InP cylinder, are randomly positioned and oriented in the $x y$ plane. (Bottom) Photoluminescence spectrum of a Au-coated (black) and an uncoated (red) InP cylinder, with $r_{I n P}=400 \mathrm{~nm}, h_{\text {air }}=340 \mathrm{~nm}$, and $h_{I n P}=260 \mathrm{~nm}$, obtained under highintensity continuous excitation; the cavity resonance can be clearly seen at $1325 \mathrm{~nm}$. Noise in the $[1350 \mathrm{~nm}$ to $1450 \mathrm{~nm}]$ window is due to strong absorption by $\mathrm{H}_{2} \mathrm{O}$ and $\mathrm{CO}_{2}$ in the atmosphere. Both curves are arbitrarily normalized to unity at $\lambda=1375 \mathrm{~nm}$.
The 260-nm-thick InP/QD/InP stack was grown on a $1 \mu \mathrm{m}$-thick sacrificial InGaAs layer by metal-organic chemical vapor deposition (MOCVD). ${ }^{25-27}$ Electron-beam lithography was used to define the position and diameter of the InP nano-cylinders that were subsequently etched by a $\mathrm{HBr} / \mathrm{O}_{2}$ mixture in an Inductively Coupled Plasma Reactive Ion Etching reactor. A thin $15 \mathrm{~nm}$-thick SiN layer was deposited around the cylinders to isolate the semiconductor from the subsequent metallic coating. A $10 \mathrm{~nm}$-thick Ti layer was deposited on top of the SiN layer, to enhance adhesion and uniformity of the gold layer to be deposited subsequently. Gold was deposited, first by sputtering, then by electrolysis, until an $80 \mu \mathrm{m}$-thick layer was formed. The InP substrate and InGaAs sacrificial layers were then chemically removed.

The modification of the spontaneous emission rate was investigated by time-resolved microphotoluminescence measurements. The samples were placed in a liquid He flow cryostat at $4 \mathrm{~K}$ and were excited optically under pulsed excitation with 150 -fs-long pulses at $80 \mathrm{MHz}$ repetition rate, delivered by a Ti:Sa laser emitting at $840 \mathrm{~nm}$. The excitation pulses were focused on the samples by a microscope objective (Mitutoyo, numerical aperture of 0.4 ) to a $2 \mu$ m-diameter spot. An incident power of $100 \mathrm{nW}$ thus corresponds to a pulse energy density of $312 \mathrm{~nJ} \mathrm{~cm}{ }^{-2}$. The QD luminescence was collected by the same microscope objective and separated from the pumping laser by means of a dichroic mirror. Residual pump photons were absorbed by a broadband antireflection-coated Si filter. The spontaneous emission was spectrally dispersed by a 0.5 -m spectrometer and detected either by a cooled InGaAs photodiode array (Roper Scientific) or by time-resolved superconducting single photon counters (SCONTEL) with a timing resolution of $50 \mathrm{ps.}$

Figure 2 presents microphotoluminescence decay transients of two QDs that are on-resonance (green curve) and offresonance (red curve) with the cavity mode, as well as the intrinsic quantum dot dynamics measured on an uncoated mesa (black curve). These curves were obtained at an excitation power of $\mathrm{P}=0.5 \mathrm{P}_{\text {sat }}$ (where $\mathrm{P}_{\text {sat }}$ is the saturation power for each QD) corresponding to a mean number of 0.5 exciton per QD and per excitation pulse. For all transients measured on 40 different single dots embedded in metal-coated nanocylinders as well as on several uncoated mesas, we observe

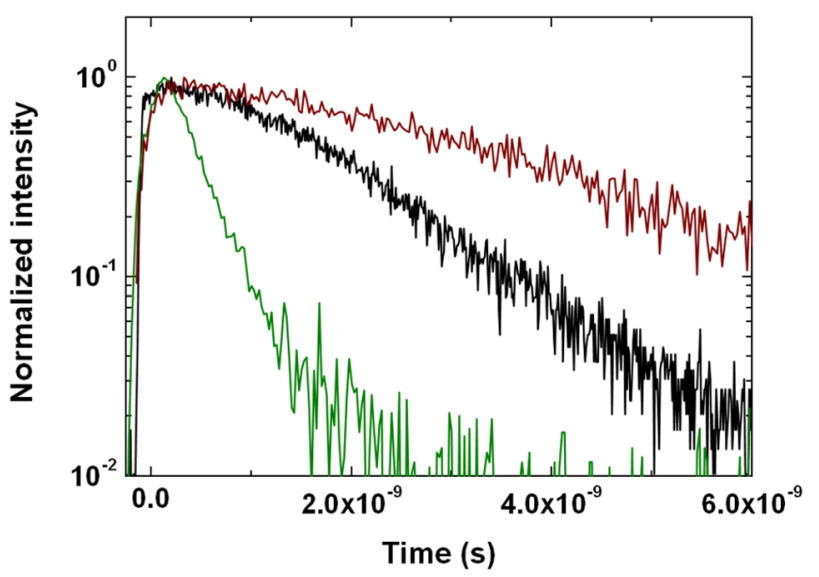

FIG. 2. Decay curves for an on- (green curve) and off- (red curve) resonance quantum dot as well as for a quantum dot in an uncoated mesa (black curve), corresponding to lifetimes of $\tau=0.33 \mathrm{~ns}, 5.2 \mathrm{~ns}$, and $1.3 \mathrm{~ns}$, respectively. 
monoexponential decays. The lifetime of single QDs in uncoated mesas was measured to be $\tau_{\text {mesa }}=1.3 \pm 0.1 \mathrm{~ns}$ for all emission lines in the spectral range from $1200 \mathrm{~nm}$ to $1400 \mathrm{~nm}$, close to that measured on unpatterned samples. ${ }^{24}$ For QDs in Au-coated nanocylinders, lifetimes ranging from $\tau_{\text {on }}=0.33 \mathrm{~ns}$ and $\tau_{\text {off }}=5.2 \mathrm{~ns}$ were measured respectively for QDs on and off resonance with the cavity mode. It should be noted that under pulsed excitation the number of photocounts collected from on- and off-resonance QDs is about the same, while under continuous excitation the on-resonance QDs display a luminescence intensity 8 to 10 times stronger than that of off-resonance dots. This implies that the shortening of the lifetime observed for on-resonance QDs is radiative and that non-radiative channels (which would occur if, for example, the QDs were too close to the metallic cavity walls) are negligible. These changes in radiative lifetimes correspond to Purcell factors $F_{p}$ ranging form $F_{p}=\tau_{\text {mesa }} / \tau_{\text {on }}$ $=3.9$ (on-resonance spontaneous emission enhancement) to $F_{p}=\tau_{\text {mesa }} / \tau_{\text {off }}=0.25$ (off-resonance inhibition).

The spontaneous emission enhancement is found to occur over a remarkably large bandwidth $(\Delta \lambda \simeq 20 \mathrm{~nm})$, thanks to the low quality factor of the cavity combined with its wavelength-sized volume. Outside this band, the QDs exhibit inhibition of the spontaneous emission over a range of $\Delta \lambda \simeq 100 \mathrm{~nm}$; this inhibition results from the detuning between the QDs and the mode of the cavity, and the absence of other modes to which the QDs could couple.

The values obtained for the Purcell factor show strong variation, even at resonance, a feature which can be explained by the random positions and orientations of the QDs, with respect to the spatially varying intra-cavity field. To confirm this interpretation, a fully vectorial computation of the spontaneous emission modification with a modal method ${ }^{9}$ was performed. Figure 3 (top) presents the expected Purcell factor
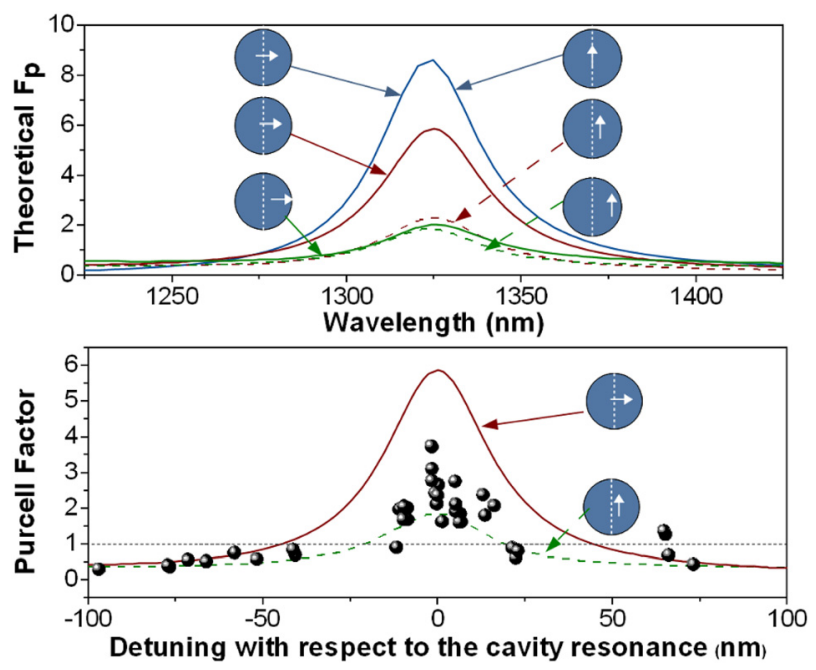

FIG. 3. (Top) Theoretical Purcell factor for the cavity under consideration as a function of wavelength. Five configurations are displayed: centered dipole (blue), dipole shifted by $100 \mathrm{~nm}$ (red), or $200 \mathrm{~nm}$ (green) from the center in either radial (solid) or ortho-radial (dotted) orientation. (Bottom) Experimental points for the modification of the spontaneous emission rate of embedded single quantum dots as a function of the detuning of the emission from the cavity resonance $(1325 \mathrm{~nm})$; the expected spontaneous emission rate modification for radially (red line) and ortho-radially (green dotted line) oriented dipoles located, respectively, $100 \mathrm{~nm}$ and $200 \mathrm{~nm}$ off-axis, are shown as indicative values for the upper and lower bounds of the experimental Purcell factors. for 3 different locations and 2 orientations of the QD dipole, taking into account the actual geometry. For an on-axis dipole the expected Purcell factor reaches its maximum value of $\mathrm{F}_{p}=9$ at resonance. However, the probability of having a QD within $50 \mathrm{~nm}$ from the axis is less than $2 \%$. On the other hand, for a QD shifted by $100 \mathrm{~nm}$ off-axis the on-resonance Purcell factor reaches $\mathrm{F}_{p}=6$ for a $\mathrm{QD}$ oriented radially, and $\mathrm{F}_{p}=2$ for an ortho-radial orientation. The variation of the Purcell factor with distance and orientation, due to the random nucleation sites of the QDs, is at the origin of the large dispersion observed for the spontaneous emission modification. Figure 3 (bottom) presents the experimental values of the Purcell factor (red dots), plotted against the QD-cavity detuning, as well as two curves representing the expected Purcell factor for radially and ortho-radially oriented QDs located, respectively, $100 \mathrm{~nm}$ and $200 \mathrm{~nm}$ from the cavity center. The radial orientation curve may be considered as representing the maximum attainable Purcell factor, since 94\% of the QDs lie outside a $100 \mathrm{~nm}$-radius disk. We note that most experimental points fall between the two curves, underscoring the random positions and orientations of the emitting dipoles. In addition, several points are within the inhibition region (with $\mathrm{F}_{p}<1$ ), below the curve for ortho-radially oriented dipoles, corresponding probably to QDs located in the proximity of the radial nodes of the cavity mode.

In conclusion, we have demonstrated a metallic cavity geometry suitable for the control of spontaneous emission of large and sparse semiconductor QDs emitting at telecommunications wavelengths. This geometry is a metal-coated semiconductor cylinder (containing the QDs) surmounted by an air-filled metallic collar, which ensures a high reflectivity for the open end of the nano-cavity. This produces a quality factor for the nano-cavity of the order of 60 and gives rise to a substantial Purcell effect even for the relatively large modal volume $\left(\simeq 0.5(\lambda / n)^{3}\right)$ necessary to enclose such emitters. These nanostructures can produce significant broadband spontaneous emission enhancement (over $\sim 20 \mathrm{~nm}$ ) and inhibition (over $\sim 100 \mathrm{~nm}$ ) in the telecommunications O-band, at $1.3 \mu \mathrm{m}$. Their performance may be improved by omitting the adhesion-enhancing Ti-layer, which is a source of optical losses, thus increasing the amplitude of the spontaneous emission enhancement (up to a few tens) while offering a significant photon collection efficiency (up to a few tens $\%)$. While the limited $Q$ factor of such cavities ( $\sim$ a few $10^{2}$ at most) is not favourable for implementing strong coupling effects (whose strength varies as $Q^{2} / V$ ), such resonators are promising candidates for tailoring the emission of large and sparse solid-state quantum emitters in the weak coupling regime, with direct implication in the development of nanoscale lasers as well as indistinguishable or entangled photon sources for quantum information technologies.

The authors acknowledge financial support from the National Network of Large Technological Facilities for Basic Technological Research (BTR).

${ }^{1}$ E. M. Purcell, Phys. Rev. 69, 37 (1946).

${ }^{2}$ P. Goy, J. M. Raimond, M. Gross, and S. Haroche, Phys. Rev. Lett. 50, 1903 (1983).

${ }^{3}$ J. M. Gérard, B. Sermage, B. Gayral, B. Legrand, E. Costard, and V. Thierry-Mieg, Phys. Rev. Lett. 81, 1110 (1998). 
${ }^{4}$ A. Kiraz, P. Michler, C. Becher, B. Gayral, A. Imamoglu, L. Zhang, E. Hu, W. V. Schoenfeld, and P. M. Petroff, Appl. Phys. Lett. 78, 3932 (2001).

${ }^{5}$ M. Bayer, T. L. Reinecke, F. Weidner, A. Larionov, A. McDonald, and A. Forchel, Phys. Rev. Lett. 86, 3168 (2001).

${ }^{6}$ D. Englund, D. Fattal, E. Waks, G. S. Solomon, B. Zhang, T. Nakaoka, Y. Arakawa, Y. Yamamoto, and J. Vuckovic, Phys. Rev. Lett. 95, 013904 (2005).

${ }^{7}$ A. Badolato, K. Hennessy, M. Atatüre, J. Dreiser, E. Hu, P. M. Petroff, and A. Imamoglu, Science 308, 1158 (2005).

${ }^{8}$ M. Larqué, I. Robert-Philip, and A. Beveratos, Phys. Rev. A 77, 042118 (2008).

${ }^{9}$ I. S. Maksymov, M. Besbes, J.-P. Hugonin, J. Yang, A. Beveratos, I. Sagnes, I. Robert-Philip, and P. Lalanne, Phys. Rev. Lett. 105, 180502 (2010).

${ }^{10}$ C. Santori, D. Fattal, J. Vuckovic, G. S. Solomon, and Y. Yamamoto, Nature 419, 594 (2002).

${ }^{11}$ A. Dousse, J. Suffczynski, A. Beveratos, O. Krebs, A. Lemaître, I. Sagnes, J. Bloch, P. Voisin, and P. Senellart, Nature 466, 217 (2010).

${ }^{12}$ O. Painter, R. K. Lee, A. Scherer, A. Yariv, J. D. O'Brien, P. D. Dapkus, and I. Kim, Science 284, 1819 (1999).

${ }^{13}$ R. Colombelli, K. Srinivasan, M. Troccoli, O. Painter, C. F. Gmachl, D. M. Tennant, A. M. Sergent, D. L. Sivco, A. Y. Cho, and F. Capasso, Science 302, 1374 (2003).

${ }^{14}$ M. Khajavikhan, A. Simic, M. Katz, J. H. Lee, B. Slutsky, A. Mizrahi, V. Lomakin, and Y. Fainman, Nature 482, 204 (2012).

${ }^{15}$ L. P. Balet, M. Francardi, A. Gerardino, N. Chauvin, B. Alloing, C. Zinoni, C. Monat, L. Li, N. Le Thomas, R. Houdré, and A. Fiore, Appl. Phys. Lett. 91, 123115 (2007).

${ }^{16}$ N. Chauvin, P. Nedel, C. Seassal, B. Ben Bakir, X. Letartre, M. Gendry, A. Fiore, and P. Viktorovitch, Phys. Rev. B 80, 045315 (2009).
${ }^{17}$ A. Dousse, L. Lancon, J. Suffczynski, E. Semenova, A. Miard, A. Lemaitre, I. Sagnes, C. Roblin, J. Bloch, and P. Senellart, Phys. Rev. Lett. 101, 267404 (2008).

${ }^{18}$ A. Surrente, M. Felici, P. Gallo, B. Dwir, A. Rudra, G. Biasiol, L. Sorba, and E. Kapon, Nanotechnology 22, 465203 (2011).

${ }^{19}$ D. Dalacu, K. Mnaymneh, V. Sazonova, P. J. Poole, G. C. Aers, J. Lapointe, R. Cheriton, A. J. SpringThorpe, and R. Williams, Phys. Rev. B 82, 033301 (2010).

${ }^{20}$ C. Belacel, B. Habert, F. Bigourdan, F. Marquier, J.-P. Hugonin, S. Michaelis de Vasconcellos, X. Lafosse, L. Coolen, C. Schwob, C. Javaux, B. Dubertret, J.-J. Greffet, P. Senellart, and A. Maitre, Nano Lett. 13, 1516 (2013).

${ }^{21}$ S. Kühn, U. Hakanson, L. Rogobete, and V. Sandoghdar, Phys. Rev. Lett. 97, 017402 (2006).

${ }^{22}$ J. T. Choy, B. J. M. Hausmann, T. M. Babinec, I. Bulu, M. Khan, P. Maletinsky, A. Yacoby, and M. Loncar, Nature Photon. 5, 738 (2011).

${ }^{23}$ B. Fain, I. Robert-Philip, A. Beveratos, C. David, Z.-Z. Wang, I. Sagnes, and J.-C. Girard, Phys. Rev. Lett. 108, 126808 (2012).

${ }^{24}$ D. Elvira, R. Hostein, B. Fain, L. Monniello, A. Michon, G. Beaudoin, R. Braive, I. Robert-Philip, I. Abram, I. Sagnes, and A. Beveratos, Phys. Rev. B 84, 195302 (2011).

${ }^{25}$ A. Michon, R. Hostein, G. Patriarche, N. Gogneau, G. Beaudoin, A. Beveratos, I. Robert-Philip, S. Laurent, S. Sauvage, P. Boucaud, and I. Sagnes, J. Appl. Phys. 104, 043504 (2008).

${ }^{26} \mathrm{O}$. Gazzano, S. Michaelis de Vasconcellos, K. Gauthron, C. Symonds, J. Bloch, P. Voisin, J. Bellessa, A. Lemaitre, and P. Senellart, Phys. Rev. Lett. 107, 247402 (2011).

${ }^{27}$ M. T. Hill, Y.-S. Oei, B. Smalbrugge, Y. Zhu, T. de Vries, P. J. van Veldhoven, F. W. M. van Otten, T. J. Eijkemans, J. P. Turkiewicz, H. de Waardt, E. J. Geluk, S.-H. Kwon, Y.-H. Lee, R. Nötzel, and M. K. Smit, Nature Photon. 1, 589 (2007). 